\title{
Nestling discrimination and feeding habits during brooding of Chestnut Thrushes
}

\author{
Tingting Yi ${ }^{1,2} \mathbb{0}$, Yue-Hua Sun ${ }^{2^{*}}$ and Wei Liang ${ }^{1 *}$ (])
}

\begin{abstract}
Background: Nestling discrimination and feeding habits during brooding are important factors affecting host selection of parasitic birds. Some host birds can avoid being parasitized by discriminating their nestlings or feeding food not suitable for parasitic nestlings. Thrushes are common medium-sized birds with widespread distribution and an open nesting habit, but they are rarely parasitized. It remains controversial whether this is due to feeding habits and/ or nestling discrimination.

Methods: In this study, we tested the nestling discrimination ability and feeding habits of Chestnut Thrushes (Turdus rubrocanus) which is distributed in China's multi-cuckoo parasitism system. Their nestling discriminability and feeding habits during brooding were studied by cross-fostering experiments and video recording to examine evolutionary restrictions on nestling discrimination and whether feeding habits are consistent with the growth of cuckoo nestlings.

Results: Our results indicate that Chestnut Thrushes using earthworms as the main brooding food can feed and maintain cuckoo nestlings and show no nestling discrimination.

Conclusions: The present study confirms that feeding habits cannot be regarded as the main factor affecting Chestnut Thrushes being rarely parasitized by cuckoos but suggests that egg rejection is likely to limit the evolution of nestling discrimination in thrushes.
\end{abstract}

Keywords: Anti-parasitic strategy, Chestnut Thrushes, Egg rejection, Feeding habits, Nestling discrimination

\section{Background}

Brood parasitic birds lay eggs in a host's nest to transfer the huge cost of caring for offspring (Davies 2000). This reproductive cost prompts the evolution of a series of anti-parasitic strategies in hosts of brood parasites, which in turn impel the evolution of new means of parasitism by the brood parasite Davies and Brooke (1989a, b); Soler 2017). For example, a host's egg recognition and rejection can stimulate evolution of its parasite's egg mimicry (Brooke and Davies 1988), so that parasitic birds and their hosts develop an arms race-like coevolutionary

\footnotetext{
*Correspondence: sunyh@ioz.ac.cn; liangwei@hainnu.edu.cn ${ }^{1}$ Ministry of Education Key Laboratory for Ecology of Tropical Islands, College of Life Sciences, Hainan Normal University, Haikou 571158, China ${ }^{2}$ Key Laboratory of Animal Ecology and Conservation Biology, Institute of Zoology, Chinese Academy of Sciences, Beijing 100101, China
}

relationship (Rothstein 1990; Davies 2011; Soler 2014). Although many hosts can accurately discriminate nonmimetic eggs of cuckoos and even highly mimicking parasitic eggs, most cuckoo hosts are unable to discriminate cuckoo nestlings that differ greatly from their own nestlings in the brooding stage (Rothstein 1982; Davies 2011). For instance, Yang et al. (2015) found that Barn Swallows (Hirundo rustica) could discriminate foreign mimicking and non-mimicking eggs but accepted all Common Cuckoo (Cuculus canorus) nestlings. Previously, it was believed that the cost of evolving nestling discriminability was too great to be a suitable defense for the host (Lotem 1993), but this hypothesis was rejected with the discovery of species with nestling discriminability (Langmore et al. 2003; Sato et al. 2010; Tokue and Ueda 2010; Noh et al. 2018; Attisano et al. 2018). Grim (2006a) summarized previous reasons for the lack of nestling discriminability 
of hosts and emphasized that egg recognition plays an important role in restricting the evolution of nestling discrimination. That is, egg rejection behavior can prevent the host from encountering parasitic nestlings, thus, weakening the evolution of its defensive measures at the nestling stage, termed the rarer enemy hypothesis. Based on this, Grim (2006a) predicted that nestling discriminability will evolve only when the host accepts all parasitic eggs (100\%). Yang et al. (2015) found that Red-rumped Swallows (Hirundo daurica) accepted all non-mimicking and mimicking eggs but rejected all Common Cuckoo (Cuculus c. bakeri) nestlings.

Although egg recognition is a common anti-parasitic strategy in most hosts (Davies and Brooke 1989a; Lovászi and Moskát 2004; Antonov et al. 2006), discrimination at the nestling stage is also likely to be an important factor restricting cuckoo parasitism (Grim 2006b; Grim et al. 2011). For instance, the Eurasian Skylark (Alauda arvensis) is an accidental cuckoo host. In a study of 348 Eurasian Skylark nests, only one was parasitized by Common Cuckoos, but the skylarks removed 3-day-old cuckoo nestlings from their nests and nurtured only their own offspring (Hegemann and Voesten 2011). In addition, Shiny Cowbird (Molothrus bonariensis) nestlings parasitizing the nests of Rufous-bellied Thrushes (Turdus rufiventris) grew poorly, and nearly $70 \%$ of the parasitic nestlings starved to death (Lichtenstein 2001). In addition to the competition between cowbird nestlings and host nestlings, nestling discrimination by the host appears to be an important factor (Lichtenstein 2001).

Apart from discrimination at the nestling stage, feeding habits are also critical in influencing host selection of cuckoos (Davies 2000; Grim et al. 2011). Soler et al. (1999) deemed that insectivorous birds with large populations and open nests were usually suitable hosts for cuckoos. Recent studies have shown that medium-sized birds were used more by cuckoos than large or small birds (Stokke et al. 2018). In Europe, thrushes such as Blackbirds (Turdus merula) and Song Thrushes (T. philomelos) have large populations, high reproductive density, open and obvious nests, and medium size, but are rarely parasitized by cuckoos (Moksnes and Røskaft 1995; Soler et al. 1999; Grim et al. 2011). In China, there is only one report that Indian Cuckoos (Cuculus micropterus) parasitized blackbirds in the wild (Zhou et al. 2001). The reason that thrushes are rarely parasitized has attracted research attention (Grim 2006a, b; Grim et al. 2011). Some studies have suggested that inconsistency with cuckoo nestling feeding habits can account for this (Davies 2000). The main food used by song thrushes to feed cuckoo nestlings was earthworms (Grim 2006b), while more frequently parasitized hosts never feed cuckoo nestlings with earthworms or food of similar size (Grim and Honza 2001).
These comparisons demonstrate that feeding habits are an important factor for thrushes rarely being parasitized, but cross-fostering experiments in Europe have also indicated that cuckoo nestlings fed alone in the nests of song thrushes can survive until fledging, and grow faster and weigh more when fledged than those fed by other hosts (Grim 2006b; Grim et al. 2011). Glue and Morgan (1972) documented thrushes feeding a common cuckoo nestling until it fledged. However, cuckoo nestlings growing in blackbird nests tended to have poor body condition, since blackbirds did not feed individual cuckoo nestlings or abandoned the nest entirely (Grim et al. 2011). Furthermore, Wood Thrushes (Hylocichla mustelina) in America are parasitized by the parasitic bird Brown-headed Cowbird (Molothrus ater) and are able to feed and maintain individual Brown-headed Cowbird nestlings (Brackbill 1958). Unlike the single-cuckoo parasitism system in Europe and the cowbird parasitism system in America, in China's multi-cuckoo parasitism system, there are as many as 17 parasitic cuckoos of different sizes (Yang et al. 2012; Zheng 2017), raising the possibility of thrushes being parasitized. The possibility of cuckoo nestling discriminability and feeding habits of thrushes should be investigated in more species, to identify those that are consistent with the growth of cuckoo nestlings.

The purpose of this study was to test the discriminability of Chestnut Thrushes (Turdus rubrocanus) for foreign nestlings in China's multi-cuckoo parasitism system. We also compared Chestnut Thrushes with a common host of Large Hawk Cuckoo (Hierococcyx sparverioides), the Elliot's Laughingthrushe (Trochalopteron elliotii) feeding their own nestlings to measure and record the growth of nestlings. A micro-camera was used to record the brooding of Chestnut Thrushes feeding cuckoo nestlings in order to document the brooding frequency of parent birds and food composition by video recordings. We predicted that Chestnut Thrushes would lack nestling discriminability and could feed and maintain foreign nestlings, which would indirectly indicate that the feeding habits of Chestnut Thrushes are suitable for the growth of cuckoo nestlings. Conversely, we predicted that if foreign nestlings can be discriminated, Chestnut Thrushes will refuse to feed them or will abandon the nest, causing the nestlings to grow poorly and fail to fledge.

\section{Methods}

\section{Study area and study species}

We conducted this study from April to August 20182019 in a farmland landscape at the northern edge of the Lianhuashan National Nature Reserve, Gansu Province, central China $\left(34.67^{\circ} \mathrm{N}, 103.50^{\circ} \mathrm{E}\right)$. The area of the nature reserve was approximately 12,000 ha, with an 
average annual temperature of $5.1-6.0{ }^{\circ} \mathrm{C}$ and an average annual rainfall of approximately $650 \mathrm{~mm}$. The altitude was approximately $2000-3500 \mathrm{~m}$, and the main peak was $3578 \mathrm{~m}$ above sea level (Sun et al. 2008). Five species of cuckoos were distributed in the same area as Chestnut Thrushes, including Common Cuckoos, Himalayan Cuckoos (Cuculus saturatus), Lesser Cuckoos (C. poliocephalus), Indian Cuckoos, and Large Hawk Cuckoos (Sun et al. 2008). The most common host of Large Hawk Cuckoos is Elliot's Laughingthrush, with a parasitism rate of $8.3 \%$ (Hu et al. 2013).

The Chestnut Thrush belongs to genus Turdus, in family Turdidae and order Passeriformes, which is of medium size and distributed throughout the Indian subcontinent, the Indochina Peninsula, and the southwest and southeast areas of China (Collar 2005). Chestnut Thrushes are one of the most abundant bird species in our study area, mainly inhabiting montane broad-leaved forests as well as coniferous and broad-leaved mixed forests (Zhao and Sun 2016) at an altitude of 2000-3500 m. The breeding period is from April to August, and its nests built from late April to early May, which are in the shape of an open cup at a height of 1-3 $\mathrm{m}$ from the ground with poor concealment (Hu et al. 2017). Nests weigh around $250 \mathrm{~g}$ with an inner diameter of $100 \mathrm{~mm}$ and an outer diameter of $140 \mathrm{~mm}$, with a cup depth of approximately $62 \mathrm{~mm}$. The egg incubation period is approximately 13 days, followed by roughly 15 days' brooding period (Zhao and Sun 2018).

\section{Cross-fostering experiments}

Since we observed no Chestnut Thrush nest parasitism by cuckoos in the study area from 2018 to 2019, we carried out cross-fostering experiments following the methods of Grim (2006b) and Grim et al. (2011). The nestlings of Elliot's Laughingthrushes and Large Hawk Cuckoos found in the nests of Elliot's Laughingthrushes were transferred to the nests of Chestnut Thrushes of the same nestling age so as to test the nestling discriminability of Chestnut Thrushes. We divided the experiments into four groups. In the first group, Chestnut Thrushes fed a Large Hawk Cuckoo nestling alone, with no other nestlings present (Fig. 1a). In the second group, Chestnut Thrushes fed two of their own nestlings and two Elliot's
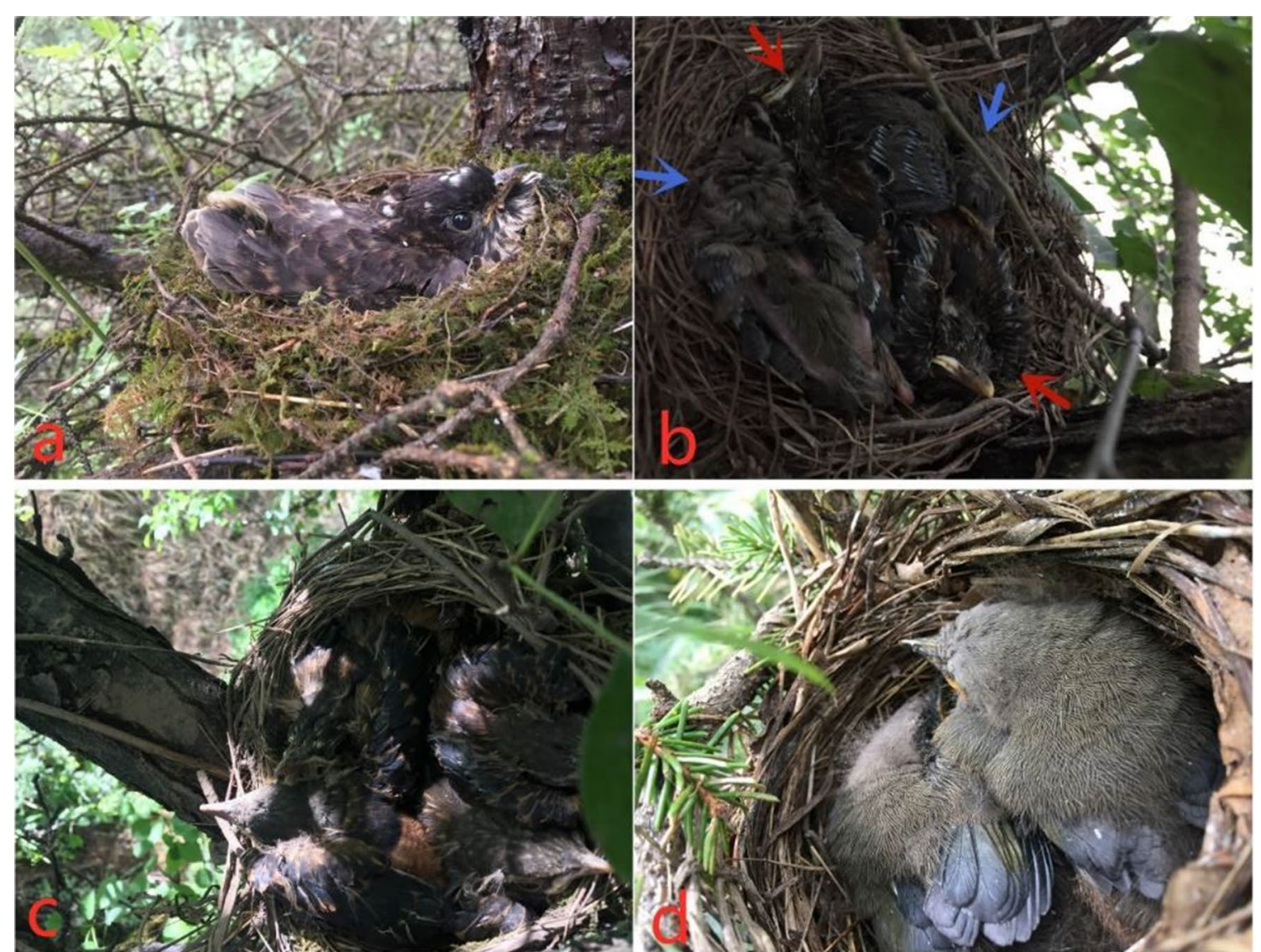

Fig. 1 Large Hawk Cuckoo nestling fed by Chestnut Thrush (a), Chestnut Thrush nestlings (red arrows) and two Elliot's Laughingthrush nestlings (blue arrows) fed by Chestnut Thrush (b), Chestnut Thrush nestlings fed by own parents (c), Elliot's Laughingthrush nestlings fed own parents 
Laughingthrush nestlings (Fig. 1b). In the third group, Chestnut Thrushes fed only their own nestlings (Fig. 1c). Finally, in the fourth group, Elliot's Laughingthrushes fed their own nestlings (Fig. 1d). The first and second groups were used to test the discriminability of Chestnut Thrushes for foreign nestlings, and the third and fourth groups served as controls for the first group. We documented and compared the growth and survival days of nestlings fed separately in the first, second, and fourth groups. Nests were checked every 2 days to observe outcomes for each nest as preyed on, fledged, or abandoned. In addition, since cuckoo nestlings sometimes push other eggs or nestlings out of the nest after hatching, we manually removed Chestnut Thrush nestlings from the experimental nests for feeding.

A WIFI/P2P miniature network camera (HD99S-32G, Shenzhen Skywork Digital Co. Ltd., Shenzhen, China) with a 20,000 mA portable battery (ROMOSS, Shenzhen Romoss Technology Co. Ltd., Shenzhen, China) as a power supply was used to record the nests of Chestnut Thrushes feeding Large Hawk Cuckoo nestlings at least once every 2 days, between 06:00 a.m. and 19:00 p.m. We analysed these recordings and documented the feeding frequency and type of food used during the brooding period. Type of food was identified to species if possible, or at minimum whether it was insects, worms, or berries. Brooding time, defined as the duration from hatching to fledging, and growth parameters (body mass and tarsus length) were measured and recorded. Body mass and tarsus length were measured using an electronic scale accurate to $0.01 \mathrm{~g}$ and a Vernier caliper accurate to $0.01 \mathrm{~mm}$. The measurement was made in the same time slots. Because nestlings of these species may leave the nest early due to human interference during the late growth period, we took final measurements at 11 days of age. Data are presented as mean \pm SD.

\section{Results}

In the experiments to test the foreign nestling discriminability of Chestnut Thrushes, Chestnut Thrushes accepted $100 \%$ of Large Hawk Cuckoo nestlings fed alone and Laughingthrush nestlings fed with own nestlings in mixed nests ( $n=3$ and $n=2$, respectively), with all nestlings fed until they fledged or were preyed upon (Table 1). When Large Hawk Cuckoo nestlings were fed alone, one nest was depredated when the nestling was 13 days old, and nestlings in the other two nests fledged at 20 and 22 days of age. In the mixed nests, Chestnut Thrushes fed two of their own nestlings and two Elliot's Laughingthrush nestlings at the same time; nestlings in one nest fledged successfully, and those in the other nest fledged early at 12 days due to human interference. In addition, Chestnut Thrushes and Elliot's Laughingthrushes in the control groups successfully fed their nestlings until fledging under natural conditions (Table 1).

Prior to 11 days of age Chestnut Thrush nestlings were heavier than large cuckoo and Elliot's Laughingthrush nestlings (Fig. 2), while Large Hawk Cuckoo nestlings were heavier than Elliot's Laughingthrush nestlings. The mass of nestlings fed by Chestnut Thrushes increased from $10.2 \mathrm{~g}(n=1)$ at 2 days of age to $102.28 \pm 4.65 \mathrm{~g}$ $(n=2)$ before fledging on day 19 (Fig. 2). Tarsus length of Large Hawk Cuckoo nestlings grew from $6.23 \mathrm{~mm}(n=1)$ to $27.75 \pm 2.00 \mathrm{~mm}(n=2)$ before fledging, shorter than that of nestlings in Chestnut Thrush and Elliot's Laughingthrush (Fig. 3).

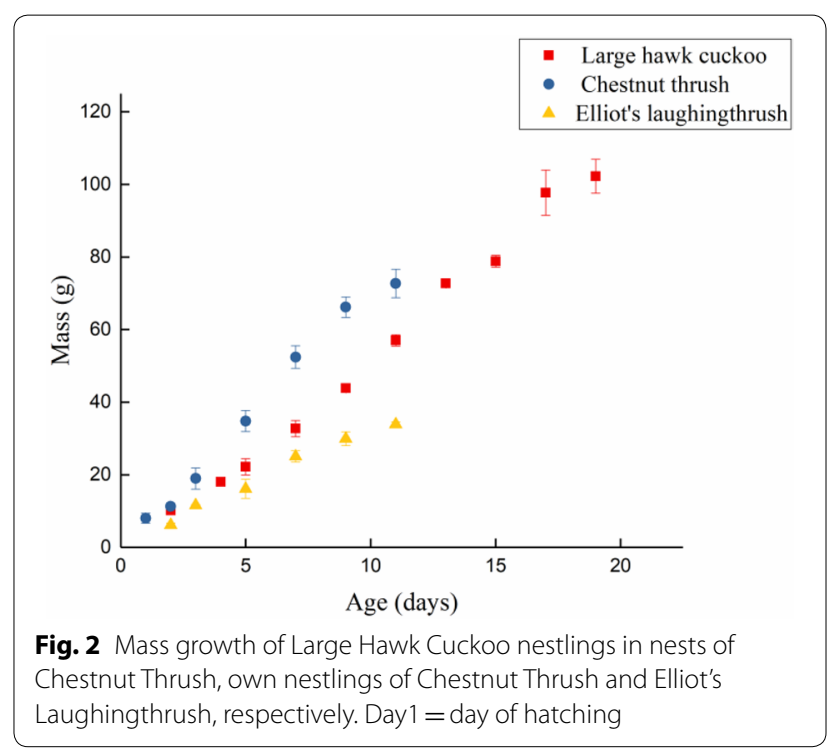

Table 1 Survival of nestlings under natural and experimental conditions

\begin{tabular}{|c|c|c|c|c|}
\hline Host & Treatment & Brood composition & Sample size (nest) & Fate of nestling \\
\hline Chestnut Thrush & Alone & One Large Hawk Cuckoo nestling & 3 & $\begin{array}{l}\text { Fledged }(n=2) \\
\text { Predated }(n=1)\end{array}$ \\
\hline Chestnut Thrush & Mixed & $\begin{array}{l}\text { Two own nestlings and two laughing thrush } \\
\text { nestlings }\end{array}$ & 2 & Fledged $(n=2)$ \\
\hline Chestnut Thrush & Natural & All own nestlings & 3 & Fledged $(n=3)$ \\
\hline Elliot's Laughing Thrush & Natural & All own nestlings & 2 & Fledged $(n=2)$ \\
\hline
\end{tabular}




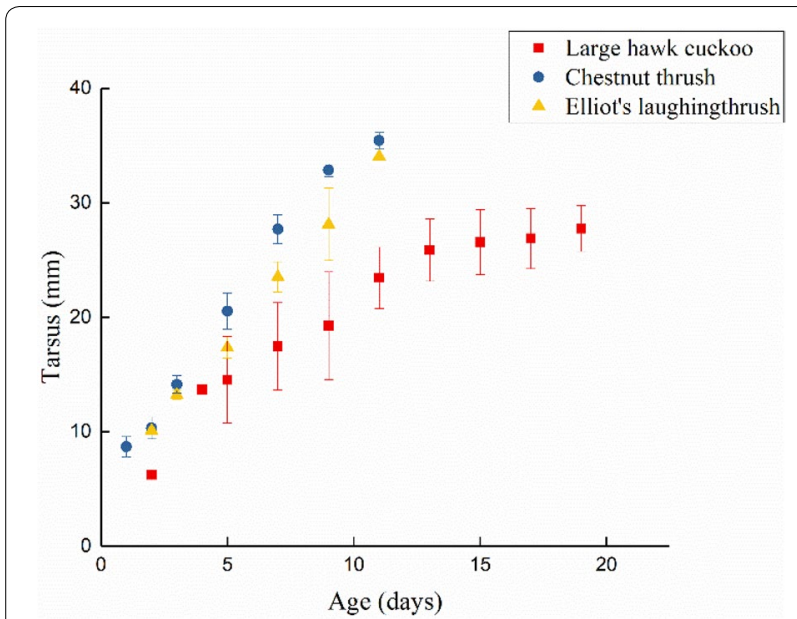

Fig. 3 Tarsus length of cuckoo nestlings in nests of Chestnut Thrush, own nestlings of Chestnut Thrush and Elliot's Laughingthrush, respectively. Day $1=$ day of hatching

We recorded videos of two nests of Chestnut Thrush parents feeding Large Hawk Cuckoo nestlings, and of female birds brooding their own nestlings and feeding Large Hawk Cuckoo nestlings to fledging. We did not obtain video of parents feeding a mixed-species nest, and the type of food could not be identified exactly because the videos were black and white. Based on the statistics of the feeding frequency in the three nests where Chestnut Thrushes fed Large Hawk Cuckoo nestlings, the recording time was a total of $2304 \mathrm{~min}$, and the nestlings were fed 621 times altogether. Earthworms accounted for $62.3 \%$ of items fed to nestlings, insects accounted for $12.5 \%$, green caterpillars, $7.4 \%$, and unidentifiable items $17.1 \%$. The remaining $0.97 \%$ of the food consisted of earthworms consumed by parent birds due to feeding failure.

\section{Discussion}

In this study, Chestnut Thrushes were not able to discriminate foreign nestlings. Even with only one Large Hawk Cuckoo nestling in the nest, Chestnut Thrushes would feed it, and only one parent bird was required to feed cuckoo nestlings until they fledged. In addition, Chestnut Thrushes fed cuckoo nestlings mostly with earthworms, but this did not affect the growth of cuckoo nestlings. This indicated that the feeding habits of Chestnut Thrushes during brooding were suitable for the growth of cuckoo nestlings.

In the brooding stage, nestling discrimination plays a prominent part in the co-evolutionary relationship of brood parasitism (Soler 2009). In American Rufous-bellied Thrushes, non-evicting parasitic birds such as cowbirds help drive the evolution of nestling discrimination, as the hosts can compare own and foreign nestlings (Lichtenstein 2001). In our study area, cuckoos are evictors, and cuckoo nestlings quickly push eggs or nestlings out of the nest after hatching, so it is difficult for the host to evolve nestling discrimination via comparison. In reviewing the hypotheses posited for lack of nestling discrimination, Grim (2006a) emphasized that egg discrimination plays a critical limiting role in the evolution of nestling discrimination. If a host evolves strong egg recognition, it may not have the opportunity to see parasitic nestlings and evolve nestling discrimination. Our study indicates that Chestnut Thrushes do not have the ability to discriminate nestlings, which had a rejection rate of $54 \%$ for non-mimetic model eggs (Yi T, Sun Y-H, Liang W, unpublished data; also see Yang et al. 2019; Zhang et al. 2019 for the Grey-backed Thrush Turdus hortulorum), thereby restricting their evolution of nestling discriminability.

In addition to nestling discrimination, feeding habits of host birds are a significant factor influencing host selection by cuckoos. Cuckoos are insectivorous passerine birds (Davies 2000), and many birds not used as cuckoo hosts utilize a large amount of plant-based food items such as seeds or berries during their brooding stage (Davies and Brooke 1989b; Moksnes et al. 1991; Liu et al. 2019). Furthermore, items used by song thrushes to feed cuckoo nestlings primarily include earthworms, insects, and mollusks (Grim 2006b), whereas more frequently used hosts never feed cuckoo nestlings with earthworms or food of similar size (Grim and Honza 2001). The comparison of feeding habits of thrushes with those of common hosts could explain why the feeding habits of thrushes are a vital reason for thrushes rarely being parasitized. Nevertheless, the results of this study do indicate that Chestnut Thrushes feeding nestlings a diet consisting mainly of earthworms can successfully maintain cuckoo nestlings. European song thrushes feeding and maintaining cuckoo nestlings also mainly used earthworms (Grim 2006b; Grim et al. 2011), consistent with our results. Therefore, we suggest that feeding habits are not the main reason for limiting cuckoo parasitism of Chestnut Thrushes. However, the results were inconsistent with the results of Blackbirds feeding cuckoo nestlings. In these experiments, cuckoo nestlings eventually died due to lack of feeding or nest abandonment (Grim 2006b; Grim et al. 2011). It is possible that Blackbirds refused to feed nestlings or abandoned the nest because it took longer to feed cuckoo nestlings than to feed their own (Grim 2006b). However, in our study, even though the duration of cuckoo nestling feeding was longer than the duration of feeding their own offspring, Chestnut 
Thrushes did not refuse to feed or abandon the nest in the late brooding stage.

By contrast, a Large Hawk Cuckoo nestling fed by Chestnut Thrushes fledged at the same time as nestlings parasitizing the nest of an Elliot's Laughingthrush (Hu et al. 2013). The brooding period in another nest was longer and the nestling fledged later, possibly because there was only one parent bird feeding the nestling. Although the sample size was small, this indicated that even one parent bird can successfully rear cuckoo nestlings. Our study demonstrated that the mass of Chestnut Thrush nestlings before fledging was greater than that of cuckoo and Elliot's Laughingthrush nestlings. In a study of Song Thrushes feeding their own nestlings and cuckoo nestlings, a cuckoo nestling pushed Song Thrush nestlings out of the nest at the age of 7 days (Grim et al. 2011). In general, a cuckoo nestling will push other eggs or nestlings out of the nest within 3-4 days after hatching (Grim et al. 2009). If Chestnut Thrush nestlings are heavier than the cuckoo at the time when the cuckoo would normally evict host nestlings, it may be difficult for the cuckoo nestling to exclude them, or the cuckoo nestling may grow poorly due to physical competition (Moskát and Hauber 2010). Such case would preclude successful parasitism on Chestnut Thrushes, but this will require further study.

In summary, our results indicate that Chestnut Thrushes lack nestling discriminability and can rear individual cuckoo nestlings to fledging. The reasons why they are rarely parasitized by cuckoos remain unclear, and will require further study into egg recognition, aggressiveness, and nest structure of thrushes, as well as whether nestling competition influences cuckoo nestlings pushing out eggs or nestlings from the nest.

\section{Conclusions}

The present study confirms that Chestnut Thrushes supplying earthworms as the main nestling food can rear parasitic cuckoo nestlings and that they lack the ability to discriminate foreign nestlings. Based on this, the feeding habits of Chestnut Thrushes cannot be regarded as the main factor affecting them being rarely parasitized, and the lack of nestling discrimination of Chestnut Thrushes may be related to their egg recognition, thereby limiting the evolution of nestling discrimination.

\section{Acknowledgements}

We are grateful to two anonymous reviewers for their helpful comments. We would like to thank Lianhuashan National Nature Reserve for their help and cooperation, and Yongke Zhu, Yingqiang Lou and Yun Fang for their assistance with field work.

\section{Authors' contributions}

WL and Y-HS designed the study; TY carried out field experiments and performed data analyses; TY and WL wrote the draft manuscript, and Y-HS helped improve the manuscript. All authors read and approved the final manuscript

\section{Funding}

This work was funded by the National Natural Science Foundation of China (Nos. 31772453 and 31970427 to WL, and 31472012 to Y-HS).

\section{Availability of data and materials}

The datasets used in the present study are available from the corresponding author on request.

\section{Ethics approval and consent to participate}

The experiments reported here comply with the current laws of China. Fieldwork was carried out under the permission from Lianhuashan National Nature Reserve, Gansu, China. Experimental procedures were in agreement with the Animal Research Ethics Committee of Hainan Provincial Education Centre for Ecology and Environment, Hainan Normal University (permit no. HNECEE-2014-005).

\section{Consent for publication}

Not applicable.

\section{Competing interests}

The authors declare that they have no competing interests. The funders had no role in study design, data collection and analysis, decision to publish, or preparation of the manuscript.

Received: 20 December 2019 Accepted: 12 May 2020

Published online: 20 May 2020

\section{References}

Antonov A, Stokke BG, Moksnes A, Røskaft E. Coevolutionary interactions between common cuckoos and corn buntings. Condor. 2006;108:414-22.

Attisano A, Sato NJ, Tanaka KD, Okahisa Y, Kuehn R, Gula R, et al. Visual discrimination of polymorphic nestlings in a cuckoo-host system. Sci Rep. 2018;8:10359.

Brackbill H. Nesting behavior of the wood thrush. Wilson Bull. 1958;70:70-89. Brooke ML, Davies NB. Egg mimicry by cuckoos Cuculus canorus in relation to discrimination by hosts. Nature. 1988;335:630-2.

Collar NJ. Family Turdidae (thrushes). In: del Hoyo J, Elliott A, Christie D, editors. Handbook of the birds of the world, vol. 10: Cuckoo-shrikes to thrushes. Barcelona: Lynx Edicions; 2005. p. 514-807.

Davies N, Brooke ML. An experimental study of co-evolution between the cuckoo, Cuculus canorus, and its hosts. I. Host egg discrimination. J Anim Ecol. 1989a;58:207-24.

Davies NB, Brooke ML. An experimental study of co-evolution between the cuckoo, Cuculus canorus, and its hosts. II. Host egg markings, chick discrimination and general discussion. J Anim Ecol. 1989b;58:225-36.

Davies NB. Cuckoos, cowbirds and other cheats. London:T. \& A.D. Poyser; 2000.

Davies NB. Cuckoo adaptations: trickery and tuning. J Zool. 2011;284:1-14.

Glue D, Morgan R. Cuckoo hosts in British habitats. Bird Study. 1972;19:187-92.

Grim T, Honza M. Differences in behaviour of closely related thrushes (Turdus philomelos and T. merula) to experimental parasitism by the common cuckoo Cuculus canorus. Biologia. 2001;56:549-56.

Grim T. The evolution of nestling discrimination by hosts of parasitic birds: why is rejection so rare? Evol Ecol Res. 2006a;8:785-802.

Grim T. Cuckoo growth performance in parasitized and unused hosts: Not only host size matters. Behav Ecol Sociobiol. 2006b;60:716-23.

Grim T, Rutila J, Cassey P, Hauber ME. The cost of virulence: an experimental study of egg eviction by brood parasitic chicks. Behav Ecol. 2009;20:1138-46.

Grim T, Samas P, Moskát C, Kleven O, Honza M, Moksnes A, Røskaft E, Stokkle BG. Constraints on host choice: why do parasitic birds rarely exploit some common potential hosts? J Anim Ecol. 2011;80:508-18.

Hegemann A, Voesten R. Can skylarks Alauda arvensis discriminate a parasite nestling? Possible case of nestling cuckoo Cuculus canorus ejection by its host parents. Ardea. 2011;99:117-20. 
Hu Y, Wang X, Chang H, Sun Y-H. Brood parasitism on Elliot's laughingthrush by large hawk cuckoo. Chin J Zool. 2013;48:292-3.

Hu Y, Zhao Q, Lou Y, Chen L, González MA, Sun Y-H. Parental attendance of chestnut thrush reduces nest predation during the incubation period: compensation for low nest concealment? J Ornithol. 2017;158:1111-7.

Langmore NE, Hunt S, Kilner RM. Escalation of a coevolutionary arms race through host rejection of brood parasitic young. Nature. 2003;422:157-60

Lichtenstein G. Low success of shiny cowbird chicks parasitizing rufous-bellied thrushes: chick-chick competition or parental discrimination? Anim Behav. 2001;61:401-13.

Liu J, Yang C, Liang W. Brood parasitism of rosefinches by cuckoos: suitable host or accidental parasitism? J Ethol. 2019;37:83-92.

Lotem A. Learning to recognize nestling is maladaptive for cuckoo Cuculus canorus hosts. Nature. 1993;362:743-4.

Lovászi P, Moskát C. Break-down of arms race between the red-backed shrike (Lanius collurio) and common cuckoo (Cuculus canorus). Behaviour. 2004;141:245-62.

Moksnes A, Røskaft E, Braa AT. Rejection behavior by common cuckoo hosts towards artificial brood parasite eggs. Auk. 1991:108:348-54.

Moksnes A, Røskaft E. Egg-morphs and host preference in the common cuckoo (Cuculus canorus): an analysis of cuckoo and host eggs from European museum collections. J Zool. 1995;236:625-48.

Moskát C, Hauber ME. Chick loss from mixed broods reflects severe nestmate competition between an evictor brood parasite and its hosts. Behav Proc. 2010;83:311-4.

Noh HJ, Gloag R, Langmore NE. True recognition of nestlings by hosts selects for mimetic cuckoo chicks. Proc R Soc London B Biol Sci. 2018;2018(285):20180726

Rothstein SI. Successes and failures in avian egg and nestling recognition with comments on the utility of optimal reasoning. Am Zool. 1982;22:547-60.

Rothstein SI. A model system for coevolution: avian brood parasitism. Ann Rev Ecol Syst. 1990;21:481-508.

Sato NJ, Tokue K, Noske RA, Mikami OK, Ueda K. Evicting cuckoo nestlings from the nest: a new anti-parasitism behaviour. Biol Lett. 2010;6:67-9.
Soler M. Avian brood parasitism: behaviour, ecology, evolution and coevolution. Cham, Switzerland: Springer; 2017

Soler JJ, Møller AP, Soler M. A comparative study of host selection in the European cuckoo Cuculus canorus. Oecologia. 1999;118:265-76.

Soler M. Co-evolutionary arms race between brood parasites and their hosts at the nestling stage. J Avian Biol. 2009;40:237-40.

Soler M. Long-term coevolution between avian brood parasites and their hosts. Biol Rev. 2014;89:688-704.

Stokke BG, Ratikainen II, Moksnes A, Røskaft E, Schulze-Hagen K, Leech DI, et al. Characteristics determining host suitability for a generalist parasite. Sci Rep. 2018:8:6285.

Sun Y-H, Fang Y, Klaus S, Martens J, Scherzinger W, Swenson JE. Nature of the Lianhuashan natural reserve. Shenyang: Liaoning Sci-Tech Press; 2008.

Tokue K, Ueda K. Mangrove Gerygones Gerygone laevigaster eject Little Bronze-cuckoo Chalcites minutillus hatchlings from parasitized nests. Ibis. 2010;152:835-9.

Yang C, Liang W, Antonov A, Cai Y, Stokke BG, Fossøy F, et al. Diversity of parasitic cuckoos and their hosts in China. Chin Birds. 2012;3:9-32.

Yang C, Wang L, Chen M, Liang W, Møller AP. Nestling recognition in redrumped and barn swallows. Behav Ecol Sociobiol. 2015;69:1821-6.

Yang C, Wang L, Liang W, Møller AP. High egg rejection rate in a Chinese population of grey-backed thrush. Zool Res. 2019;40:226-30.

Zhang J, Shi J, Deng W, Liang W. Nest defense and egg recognition in the grey-backed thrush (Turdus hortulorum): defense against interspecific or conspecific brood parasitism? Behav Ecol Sociobiol. 2019;73:148.

Zhao Q, Sun Y-H. Behavioral plasticity is not significantly associated with head volume in a wild chestnut thrush (Turdus rubrocanus) population. Avian Res. 2016;7:12.

Zhao Q, Sun Y-H. Nest-site characteristics and nesting success of the chestnut thrush. Ornithol Sci. 2018;17:3-9.

Zheng G. A checklist on the classification and distribution of the birds of the world (Third Edition). Beijing: Science Press; 2017.

Zhou L, Song Y, Ma Y. Studies on breeding ecology of the balckbird. Chin J Zool. 2001;20:32-4.
Ready to submit your research? Choose BMC and benefit from:

- fast, convenient online submission

- thorough peer review by experienced researchers in your field

- rapid publication on acceptance

- support for research data, including large and complex data types

- gold Open Access which fosters wider collaboration and increased citations

- maximum visibility for your research: over 100M website views per year

At BMC, research is always in progress.

Learn more biomedcentral.com/submissions 